\title{
Ocular gene therapy: introduction to the special issue
}

\author{
Gene Therapy (2012) 19, 119-120; doi:10.1038/gt.2011.189
}

It has been less than two decades since the publication of the first papers to describe gene transfer to the adult mouse eye. Since then ocular gene therapy has made rapid progress, with the first clinical trials to show clinical benefit occurring in 2008. As well as a wide range of programmes to develop gene therapy for relatively rare disorders such as Leber congenital amaurosis (LCA), there are major attempts to develop gene therapy to treat common ocular conditions such as glaucoma and age-related macular degeneration (AMD). From a handful of academic laboratories, there are now dozens of groups engaged in ocular gene therapy, including small biotechs and major pharmaceutical companies. In the field of gene therapy, the eye has now moved to centre stage. The reason for this increase in interest is perhaps an increasing recognition that the eye is an attractive target for gene therapy.

It is now clear that ocular tissues can be very efficiently and stably transduced. Over the last 20 years, many different gene delivery approaches, using a wide variety of non-viral and viral vectors, have been evaluated in the eye. The most promising vectors to date are those based on adenoviral vectors (AAV) and lentiviruses. Both types of vector are currently being used in clinical trials of ocular gene therapy and are reviewed in this special issue. ${ }^{1,2}$ Lentiviral vectors are very efficient for transducing cells in the anterior chamber and the retinal pigment epithelium, but they are much less efficient at transducing photoreceptors. AAV vectors (especially AAV8) are currently the most promising vehicles for gene delivery to the retina and photoreceptors in particular. Identification of a large number of AAV variants has further enhanced the utility of $A A V$ as a vector platform. Many of these variants have unique transduction characteristics that might be used to target different cell types in the retina. New AAV serotypes are still being characterised and new vectors, with increased infectivity, generated by mutating capsid surface tyrosines. This special issue contains a report showing that use of a novel, double-tyrosine mutant AAV9 can significantly enhance retinal gene delivery. ${ }^{3}$ It still remains to be seen how much more effective the various tyrosine mutants and different serotypes are in large animal models, including non-human primates. One further issue that is yet to be resolved is whether there are effective ways to increase the packaging capacity of $\mathrm{AAV}$ vectors in order to deliver a large number of genes to photoreceptors.

Compared with most other organ systems, the eye is small and compartmentalised, allowing the localised delivery of relatively modest amounts of gene therapy vector at or near the target site. The compartmentalised anatomy and the presence of the blood-retina barrier limits the extent of vector dissemination outside the eye and reduce the severity of immune responses to immunogenic gene transfer vectors. Another advantage for ocular gene therapy, and retinal gene therapy in particular, is that the target cell populations are typically very stable. The lack of cell division allows the use of nonintegrating vector systems for sustained transgene expression. As insertion of the vector genome into the host cell genome is associated with insertional mutagenesis and oncogenesis, the use of non-integrating systems could substantially reduce the possibility of malignant transformation of the target cells. This special issue also contains a report showing that any insertional events arising from subretinal delivery AAV and HIV-1 vectors do not have an effect upon the incidence of ocular neoplasia in $p 53$ tumour-suppressor gene knockout mice, which are highly susceptible to intraocular malignant transformation. ${ }^{4}$ These findings support the continued development of these vectors for ocular applications.

Over the last 20 years, gene therapy has been developed for a wide variety of ocular disorders from corneal dystrophies to retinoblastoma. The most advanced programmes are those concerned with gene therapy for inherited retinal disorders (IRDs). These form a large group of genetically and phenotypically heterogeneous diseases that are characterised by progressive loss of photoreceptor cells and concomitant loss of vision. IRDs affect $\sim 1$ in 3000 people in Europe and the United States. To date, about 150 genes and a further 50 loci associated with retinal dystrophy have been identified. As reviewed in this special issue, there has been huge progress with regard to gene therapy for the treatment of recessive IRDs. ${ }^{5}$ Proof of concept for the treatment of loss-of-function disorders has been established in animal models of more than 20 different forms of degenerative retinal disease, three clinical trials for LCA2 because of RPE65-deficiency have been conducted or are still on-going, and new trials for various recessive retinal degenerations are anticipated in the near future. However, there are many challenges to be addressed before gene supplementation can become a conventional treatment. Although the initial clinical trials of gene therapy for LCA2 have demonstrated safety and some clinical benefit, there is still a requirement for improved efficacy; the level of rescue is not yet as effective as that in the animal models. Effective treatments for most of the amenable disorders-particularly those affecting the retinal pigment epithelium and the more stationary photoreceptor defects-might be achieved within the next decade. However, the development of therapies for many other forms of retinal dystrophy will probably depend on overcoming a number of different challenges, such as the limited packaging capacity of AAV, and may require more appropriate control of transgene expression.

For dominant IRDs the very high levels genetic heterogeneity represents a barrier to the development of therapies focused on correcting the primary genetic defect. More than 60 different genes, with over 150 different mutations in the rhodopsin gene alone, have been implicated. Nevertheless, as reviewed in this special issue, there has been major progress in the last few years with regard to gene therapy for dominant disorders. ${ }^{6}$ So-called 'suppression and replacement' strategies involving small interfering RNA-based strategies to ablate mRNA from specific target genes in combination with delivery of resistant replacement transgenes appear to be very promising. Alternative gene therapy approaches for dominant retinitis 
pigmentosa include the modulation of oxidative stress, the provision of neurotrophic and anti-apoptotic factors to modulate apoptotic pathways and the modulation of the cellular response to the presence of aggregated proteins. Such neuroprotective strategies, aimed at delaying or halting retinal ganglion cell loss would also be valuable to save vision in glaucoma and this special issue contains a review of the progress that has been made in the use of gene therapy to understand mechanisms underlying retinal ganglion cell degeneration and to promote the survival of these neurons in experimental models of optic nerve injury. ${ }^{7}$

In recent years, there has been major interest in the development of gene therapy to treat ocular neovascularisation, which is associated with diabetic retinopathy and AMD, some of the most common ocular disorders. Gene transfer of anti-angiogenic proteins has the potential to provide long-term suppression of neovascularisation and/ or excessive vascular leakage in the eye compared with repeat intraocular administration of anti-vascular endothelial growth factor (VEGF) antibodies that is effective, but very expensive. This special issue contains a review of the studies in animal models of ocular neovascularization that have demonstrated impressive results with a number of transgenes. ${ }^{8}$ Two industry sponsored clinical trials, one using an AAV vector to express a VEGF-binding protein and another using a lentiviral vector to express endostatin and angiostatin, have started recently. These approaches may eventually take gene therapy into mainstream clinical practice.

Patients with advanced retinal degeneration have lost most of their photoreceptor cells and, therefore, all of the gene therapy strategies discussed above would be ineffective. An alternative approach that has been reviewed in this special issue is the use of optogenetics to target genetically encoded light sensors to the cells in the remaining retinal circuitry, thus converting these cells into artificial photoreceptors. ${ }^{9}$ If the artificial photoreceptors are connected to other cell types in the retinal circuit, light would also modulate the activity of these cells. The current challenges lie in choosing the right sensor and targeting strategy, so that the light-evoked retinal activity arising from artificial photoreceptors will be similar to the activity of normal retinas stimulated through normal photoreceptors. Further developments for enhancing efficacy include developing more-sensitive artificial photoreceptors that are at least as fast as normal photoreceptors, sensors that work at near-infrared wavelengths, highly effective dendritic localisation signals and vectors (perhaps new AAV serotypes) that are able to transduce bipolar cells effectively.

In this special issue, we have covered just a small part of a wide range of gene therapy approaches for the treatment of ocular disorders that are currently being explored. We have not covered, for instance, the progress in gene therapy for the treatment of corneal disease, including graft rejection and the treatment of inflammatory disorders such as uveitis. Both of these areas have tremendous potential as we gain better understanding of ocular immunology. With the development of effective vectors for transducing ocular tissues, the main limitation for effective application of this technology is sufficient understanding of disease mechanisms to enable us to design effective strategies. The ultimate prize in ocular gene therapy would be effective treatment of dry AMD, the most common cause of blindness in the developed World. Perhaps in the next decade, with increased understanding of disease mechanism, we will see trials of gene therapy to treat this disorder, for example, through modulation of the alternative complement pathway. Although it is difficult to predict when ocular gene therapy will have a major impact in the clinic, rapid progress over the last two decades suggests that it is likely to be one of the first gene therapies to do so.

\section{CONFLICT OF INTEREST}

The author is a consultant for ReGenX.

\section{ACKNOWLEDGEMENTS}

Robin Ali is a Senior Investigator, UK National Institute of Health Research

RR Ali

Department of Molecular Therapy, Institute of Ophthalmology, University College London, London, UK E-mail: r.ali@ucl.ac.uk

1 Vandenberghe LH, Auricchio A. Novel adeno-associated viral vectors for retinal gene therapy. Gene Therapy 2012; 19: 162-168.

2 Balaggan KS, Ali RR. Ocular gene delivery using lentiviral vectors. Gene Therapy 2012; 19: 145-153.

3 Dalkara D, Byrne LC, Lee T, Hoffmann NV, Schaffer DV, Flannery JG. Enhanced gene delivery to the neonatal retina through systemic administration of Tyrosine-mutated AAV9. Gene Therapy 2012; 19: 176-181.

4 Balaggan KS, Duran Y, Georgiadis A, Thaung C, Barker SE, Buch PK et al. Absence of ocular malignant transformation after sub-retinal delivery of rAAV2/2 or integrating lentiviral vectors in p53-deficient mice. Gene Therapy 2012; 19: 182-188.

5 Smith AJ, Bainbridge JWB, Ali RR. Gene supplementation therapy for recessive forms of inherited retinal dystrophies. Gene Therapy 2012; 19: 154-161.

6 Farrar GJ, Millington-Ward S, Chadderton N, Humphries P, Kenna PF. Gene-based therapies for dominantly inherited retinopathies. Gene Therapy 2012; 19: 137-144.

7 Wilson Am, DiPolo A. Gene therapy for retinal ganglion cell neuroprotection in glaucoma. Gene Therapy 2012; 19: 127-136.

8 Campochiaor PA. Gene transfer for ocular neovascularization and macular edema. Gene Therapy 2012; 19: 121-126.

9 Busskamp V, Picaud S, Sahel JA, Roska B. Optogenetic therapy for retinitis pigmentosa. Gene Therapy 2012; 19: 169-175. 